\title{
Solving Continual Two-Stage Problems of Optimal Partition of Sets
}

\author{
Zelentsov D. ${ }^{1}$, Us S. ${ }^{1}$, Koryashkina L. ${ }^{1}$, Stanina $0 .{ }^{2}$ \\ ${ }^{I}$ Department of System Analysis and Management, National Mining University, Dnipro, Ukraine \\ ${ }^{2}$ Department of Information Systems, Ukrainian State University of Chemical Technology, Dnipro, Ukraine \\ *Corresponding Author: Stanina O., Department of Information Systems, Ukrainian State University of \\ Chemical Technology, Dnipro, Ukraine
}

\begin{abstract}
The paper considers two-stage location-allocation problem being a certain case of a continual multistage problem of optimal partition of sets with the additional couplings. It has been demonstrated that solution of two-stage optimal partition of sets with restrictions to the capacities of enterprises being arranged is reduced to the minimization of nonsmooth function of finite quantity of variables with simultaneous solving a problem of linear programming of a transport type. The developed numerical algorithm synthesizing both Shor's algorithm and a method of potentials has been described. The results of model problems solution have been represented.
\end{abstract}

Keywords: optimal partition of sets, location-allocation problems, multistage partition problems, multistage location problems

\section{INTRODUCTION}

Multistage location-allocation problems are generalization of multistage transportation-production problems. A number of papers concern their analysis. The papers involve various models of such problems representing methods and algorithms to solve them. The majority of mathematical models of the problems corresponds the situation when the number of possible location places to arrange the enterprises is either infinite or their possible regional location is predetermined. Such authors as V.L. Beresnev, E.Kh. Gimadi, Yu.A. Kochetov, V.A. Trubin, D.B. Yudin and others who dealt with discrete multistage problems are worth mentioning [1, 6-9]. Numerous papers addressing to discrete location-allocation problems concerns the application of heuristic algorithms, particularly algorithms of a genetic type. However, practical solution of logistic problems may result in such situations when possible location areas of enterprises are not limited by a final set of alternatives; i.e. the enterprises may be located in any point of the determined area. Various mathematical schools are engaged in the development of mathematical models and methods to solve such problems [1-4, 6-9].

The paper is a sequential continuation of studies connected with the analysis of features of continual multistage problems of optimal partition of sets (OPS) and their solving. For the first time, mathematical models of such problems were represented in [5]. They are two-stage continual OPS problems with type 1 location of centres; they take into consideration the fact that enterprises of stage 1 should be connected with enterprises of stage 2. The latter has stipulated another name of such problems i.e. continual OPS problems with additional couplings.

\section{OBJECTIVE}

Objectives of the paper are the development of a method and solution algorithm for continual problems of optimal partition of sets, if additional coupling and integral limitations in the form of equalities are available and if effect of additional couplings on the boundaries between subsets in the context of Continual OPS problems is demonstrated.

\section{Materials \& Methods}

It is possible to formulate the description of a problem of optimal partition of sets with additional couplings in such a way [5]. Let certain enterprise exist. It is connected with subjects meant to 
accumulate (get) raw material out of suppliers distributed continually within certain area $\Omega$, process it and send for commercialization (or further processing) into the predetermined places. We will designate the enterprises processing the raw material as places of primary processing or enterprises of stage 1; we will designate places of further processing as stage enterprises. Assume also that:

- the capacity of $j^{\text {th }}$ enterprise of stage 2 is known being equal to $b_{j}^{I I}, j=1,2, . ., M$;

- resource reservoir in each point of $\Omega$ area has been estimated; it is described with the help of $\rho(x)$ function;

- a technique for cost calculation of the resource unit delivery is known $c_{i}^{I}\left(x, \tau_{i}^{I}\right), i=1,2, \ldots, N-$ from $x$ point to a point of primary processing $\tau_{i}^{I}$;

- transportation cost of a product unit $c_{i j}^{I I}\left(\tau_{i}^{I}, \tau_{j}^{I I}\right)$ from a point of primary processing $\tau_{i}^{I}$ to a $\tau_{j}^{I I}$ point is proportional to distance between the points; and

- each supplier of raw material $x \in \Omega$ is only connected with one enterprise of stage $1 \tau_{i}^{I}, i=\overline{1, N}$; in turn the latter should be connected at least with one enterprise of stage $2 \tau_{j}^{I I}, j=\overline{1, M}$.

Moreover, we assume that the capacity of $i^{\text {th }}$ manufacturer of stage 1 is determined by means of total resource reservoir within the area being serviced by the enterprise and its profits depend on transportation cost only.

It is required to determine locations of enterprises of stage $1 \tau_{i}^{I}, \ldots, \tau_{N}^{I}$, service areas form them $\Omega_{1}, \Omega_{2}, \ldots, \Omega_{N}$ transportation volumes between enterprises of stages 1 and $2 v_{11}, \ldots, v_{N M}$ in such a way to provide minimum total cost of raw material delivery and end product delivery.

Following two-stage continual problem of partition of sets with location of centres of enterprises, belonging to stage 1 , is mathematical model for the described problem.

Problem A. it is necessary to find such a partition of $\Omega$ set within $N$ of $\Omega_{1}, \Omega_{2}, \ldots, \Omega_{N}$ subsets measured according to Lebesque (empty subsets may be among them), to determine coordinates $\tau_{i}^{I}, \ldots, \tau_{N}^{I}$ of the subsets centres as well as such values as $v_{11}, \ldots, v_{N M}$ providing minimum of the functional:

$$
\begin{aligned}
& F\left(\left\{\Omega_{1}, \ldots, \Omega_{N}\right\},\left\{\tau_{1}^{I}, \ldots, \tau_{N}^{I}\right\},\left\{v_{11}, \ldots v_{N M}\right\}\right)= \\
& =\sum_{i=1}^{N} \int_{\Omega_{i}} c_{i}^{I}\left(x, \tau_{i}^{I}\right) \rho(x) d x+\sum_{i=1}^{N} \sum_{j=1}^{M} c_{i j}^{I I}\left(\tau_{i}^{I}, \tau_{j}^{I I}\right) v_{i j},
\end{aligned}
$$

if

$$
\int_{\Omega_{i}} \rho(x) d x=\sum_{j=1}^{M} v_{i j}, i=\overline{1, N},
$$

$\sum_{i=1}^{N} v_{i j}=b_{j}^{I I}, j=\overline{1, M}$

$\left\{\Omega_{1}, \ldots, \Omega_{N}\right\} \in \sum_{\Omega}^{N}$,

$v_{i j}^{I I} \geq 0, i=1,2, \ldots, N, j=1,2, \ldots, N, \tau^{I}=\left(\tau_{1}^{I}, \tau_{2}^{I}, \ldots, \tau_{N}^{I}\right), \tau^{I} \in \Omega^{N}$.

Where $\sum_{\Omega}^{N}=\left\{\left(\Omega_{1}, \ldots, \Omega_{N}\right): \bigcup_{i=1}^{N} \Omega_{i}=\Omega, \operatorname{mes}\left(\Omega_{i} \cap \Omega_{j}\right)=\varnothing, i \neq j, i, j=1, \ldots, N\right\}$

In this context, limitations (2) and (3) express balance between capacities of enterprises belonging to stages 1 and 2; thus, following equality is the condition to solve the problem: 


$$
\int_{\Omega} \rho(x) d x=\sum_{j=1}^{M} b_{j}^{I I} \text {. }
$$

A method to solve problem A means its reformulation in terms of characteristic functions of subsets making up partition of the predetermined area $\Omega$.

Problem B. Find

$$
\min _{\lambda(\cdot), \tau^{I}, v} I\left(\lambda(\cdot), \tau^{I}, v\right),
$$

Under the limitations

$$
\begin{aligned}
& \int_{\Omega} \rho(x) \lambda_{i}(x) d x=\sum_{j=1}^{M} v_{i j}, i=\overline{1, N}, \\
& \sum_{i=1}^{N} v_{i j}=b_{j}^{I I}, j=\overline{1, M} ; \\
& \lambda(\cdot) \in \Gamma_{1}, \tau^{I}=\left(\tau_{1}^{I}, \tau_{2}^{I}, \ldots, \tau_{N}^{I}\right), \tau^{I} \in \Omega^{N}, v \in R_{N M}^{+} ;
\end{aligned}
$$

Where

$$
\begin{aligned}
& I\left(\lambda(\cdot), \tau^{I}, v\right)=\sum_{i=1}^{N} \int_{\Omega} c_{i}^{I}\left(x, \tau_{i}^{I}\right) \rho(x) \lambda_{i}(x) d x+\sum_{i=1}^{N} \sum_{j=1}^{M} c_{i j}^{I I}\left(\tau_{i}^{I}, \tau_{j}^{I I}\right) v_{i j} \\
& \Gamma_{1}=\left\{\lambda(\cdot)=\lambda_{1}(\cdot), \ldots, \lambda_{N}(\cdot)\right): \lambda_{i}(\cdot)=0 \vee 1, i=\overline{1, N}, x \in \Omega, \\
& \left.\sum_{i=1}^{N} \lambda_{i}(x)=1, x \in \Omega\right\}
\end{aligned}
$$

Following formulas have been obtained for optimal components of a vector function $\lambda(\cdot)$ with the help of binary apparatus:

$$
\lambda_{i}^{*}(x)=\left\{\begin{array}{l}
1, \text { if } x \in \Omega_{i}=\left\{x \in \Omega:\left(c_{i}^{I}\left(x, \tau_{i}^{I^{*}}\right)+\psi_{i}^{*}\right) \rho(x)=\min _{m=1, N}\left(c_{m}^{I}\left(x, \tau_{m}^{I^{*}}\right)+\psi_{m}^{*}\right) \rho(x)\right\}, \\
0 \text { in other cases, }
\end{array}\right.
$$

Where optimal solution of binary problem reduced to the form:

$$
\begin{aligned}
& G(\{\psi, \eta\})=\min _{\left\{\tau^{I}, v\right\} \in \Omega^{N} \times R_{N M}^{+}} G_{1}\left(\left\{\tau^{I}, v\right\},\{\psi, \eta\}\right)= \\
& =\min _{\left\{\tau^{\prime}, v\right\} \in \Omega^{N} \times R_{N M}^{+}}\left[\int_{\Omega} \min _{k}\left(c_{k}^{I}\left(x, \tau_{k}^{I}\right)+\psi_{k}\right) \rho(x) d x+\sum_{i=1}^{N} \sum_{j=1}^{M}\left(c_{i j}^{I I}\left(\tau_{i}^{I}, \tau_{j}^{I I}\right)-\eta_{j}-\psi_{i}\right) v_{i j}\right]+ \\
& +\sum_{j=1}^{M} \eta_{j} b_{j}^{I I} \rightarrow \max , \psi \in E_{N}, \eta \in E_{M}, i=1, N, j=1, M
\end{aligned}
$$

Is selected as $\tau^{I^{*}}, v^{*}, \psi^{*}, \eta^{*}$

If:

$$
\left(c_{i j}^{I I}\left(\tau_{i}^{I}, \tau_{j}^{I I}\right)-\eta_{j}-\psi_{i}\right) \geq 0 .
$$

Relying upon the mentioned formulas to solve the problem B, iteration algorithm has been proposed. The algorithm combines a method of potentials being applied for classical problem of linear programming of transportation type and Shor's algorithm making it possible to solve optimization problem of nonsmooth function $G_{1}\left(\left\{\tau^{I}, v\right\},\{\psi, \eta\}\right)$. 


\section{Algorithm}

0 . Put $\Omega$ area within parallelepiped $\Pi$ which sides are parallel to axis of Cartesian coordinate system; assume that $\rho(x)=0$, if $x \in \Pi \backslash \Omega$. Cover parallelepiped $\Pi$ with the help of rectangular net.

1. Let $k=0$. Prescribe initial approximation of coordinates of centres $\tau^{I}=\tau^{I(k)}$ and vector functions $\lambda(x)=\lambda^{(k)}(x)$ for all points of $x \in \Omega$ net.

2. Calculate $b_{i}^{I(k)}$ values according to the formula:

$b_{i}^{I(k)}=\int_{\Omega} \rho(x) \lambda_{i}^{(k)}(x) d x, i=\overline{1, N}$.

3. Determine initial values $v_{i j}^{(k)}, i=\overline{1, N}, j=\overline{1, M}$ solving following transportation problem with the help of a method of potentials:

$$
\begin{aligned}
& \sum_{i=1}^{N} \sum_{j=1}^{M} c_{i j}^{I I}\left(\tau_{i}^{I(k)}, \tau_{j}^{I I}\right) v_{i j} \rightarrow \min , \\
& \sum_{i=1}^{N} v_{i j}=b_{j}^{I I}, j=1,2, \ldots, M, \quad \sum_{j=1}^{M} v_{i j}=b_{i}^{I(k)}, i=1,2, \ldots, N, \\
& v_{i j} \geq 0, i=1,2, \ldots, N, j=1,2, \ldots, M .
\end{aligned}
$$

Let values of $\psi_{1}^{(l)}, \psi_{2}^{(l)}, \ldots, \psi_{N}^{(l)}$ potentials have been obtained resulting from $(l)$ steps of $l=0,1,2, \ldots$ algorithm. Describe $(l+1)^{\text {th }}$ step.

Step $(l+1)$.

4. Calculate $\lambda^{(l+1)}(x)$ values within the net nodes according to the formula:

$$
\lambda_{i}^{(l+1)}(x)=\left\{\begin{array}{l}
1, \quad \text { if }\left(c_{i}^{I}\left(x, \tau_{i}^{I(l)}\right) \rho(x)+\psi_{i}^{(l)}\right) \rho(x)=\min _{m}\left(c_{m}^{I}\left(x, \tau_{m}^{I(l)}\right)+\psi_{m}^{(l)}\right) \rho(x), \\
0 \text { in other cases, } i=\overline{1, N} .
\end{array}\right.
$$

Select values of corresponding potentials obtained during the last iteration of a method of potentials as the values of binary variables $\eta_{j}^{(k)}, j=\overline{1, M}$, and $\psi_{i}^{(k)}, i=\overline{1, N}$.

5. Calculate $v_{i j}^{(l+1)}, i=\overline{1, N}, j=\overline{1, M}$ values solving transportation problem (5) - (7) using a method of potentials. Select values of potentials corresponding to optimal solution of (5) - (7) problem as the values of binary variables $\eta_{j}^{(l+1)}, j=\overline{1, M}$, and $\psi_{i}^{(l+1)}, i=\overline{1, N}$.

6. Calculate components of $g_{G}^{\tau^{l}}(v, \psi, \eta)$ subgradient within the net nodes according to the formula:

$$
g_{G}^{\tau_{i}^{l}}=\int_{\Omega} \rho(x) g_{c^{l}}^{\tau_{i}^{l}} \lambda_{i}(x) d x+\sum_{i=1}^{N} \sum_{j=1}^{M} g_{c^{I l}}^{\tau_{i j}^{l}} v_{i j}, i=\overline{1, N}
$$

where $g_{c^{I}}^{\tau_{i}^{I}}$ is $i^{\text {th }}$ component of $2 N$-dimensional vector of generalized gradient $g_{c}^{\tau^{I}}$ of $c_{i}^{I}$ function within $\tau^{I}$ point, $g_{c^{I I}}^{\tau_{i}^{I}} \mathrm{i}^{\text {th }}$ component of $2 \mathrm{~N}$-dimensional vector of generalized $g_{c}^{\tau^{I}}$ of $c_{i j}^{I I}$ function within $\tau^{I}$ point if $\tau^{I}=\tau^{I(l)}, \psi=\psi^{(l+1)}, \lambda=\lambda^{(l+1)}, v=v^{(l+1)}, \eta=\eta^{(l+1)}$.

7. Select $h_{l}>0$ step of r-algorithm and find:

$$
\tau^{I(l+1)}=P_{I}\left(\tau^{I(l)}-h_{l} B_{l}^{\tau} g_{G}^{\tau}\left(\tau^{I(l)}, v^{(l+1)}, \psi^{(l+1)}, v^{(l+1)}\right)\right),
$$


Where $\mathrm{P}_{\Pi}$ is projector operator on $\Pi, B_{l}^{\tau}$ is operator of space transformation into pivot space $E_{2 N}$ being:

$$
\left.B_{l}^{\tau}=B_{l-1}^{\tau}\left(I+\left(\frac{1}{\alpha}-1\right) \theta_{l-1}^{\tau}\left(\theta_{l-1}^{\tau}\right)^{T}\right)\right),
$$

Where I is unity matrix of corresponding dimensionality, and $\theta_{l-1}^{\tau}$ corresponds to normalized vector of difference of two sequential sub gradients within the transformed space, i.e.:

$$
\theta_{l-1}^{\tau}=\frac{\left(B_{l-1}^{\tau}\right)^{T}\left(g_{G}^{\tau}\left(\tau^{(l)}, \psi^{(l+1)}, \eta^{(l+1)}, v^{(l+1)}\right)-g_{G}^{w}\left(\tau^{(l-1)}, \psi^{(l+1)}, \eta^{(l+1)}, v^{(l+1)}\right)\right)}{\left\|\left(B_{l-1}^{\tau}\right)^{T}\left(g_{G}^{\tau}\left(\tau^{(l)}, \psi^{(l+1)}, \eta^{(l+1)}, v^{(l+1)}\right)-g_{G}^{\tau}\left(\tau^{(l-1)}, \psi^{(l+1)}, \eta^{(l+1)}, v^{(l+1)}\right)\right)\right\|},
$$

if $\left\|\left(B_{l-1}^{\tau}\right)^{T}\left(g_{G}^{\tau}\left(\tau^{(l)}, \psi^{(l+1)}, \eta^{(l+1)}, v^{(l+1)}\right)-g_{G}^{\tau}\left(\tau^{(l-1)}, \psi^{(l+1)}, \eta^{(l+1)}, v^{(l+1)}\right)\right)\right\| \geq \varepsilon_{0}$, and $\theta_{l-1}^{\tau}=0$ in other cases. In this context, $\varepsilon_{0}$ is the accuracy of representation of zero by ECM.

8. If $\left\|\left(\tau^{(l)}, v^{(l)}, \psi^{(l)}, \eta^{(l)}\right)-\left(\tau^{(l+1)}, v^{(l+1)}, \psi^{(l+1)}, \eta^{(l+1)}\right)\right\| \leq \varepsilon, \varepsilon>0$ condition is met, then we proceed to point 9; if not, then transition to $(l+2)^{\text {th }}$ step is performed.

9. Calculate values of objective functional. End of the algorithm.

The algorithm has been described.

\section{RESUlTS \& DISCUSSION}

The developed and software-implemented algorithm has been piloted using following model problem. Let resource applying multistage production is uniformly distributed within $\Omega=\{(x, y): 0 \leq x \leq 1,0 \leq y \leq 1\}$ area. Assume that no more than four enterprises of stage 1 located within $\Omega$ area can process the resource. Refinement product is transported to two enterprises of stage 2 for its further use.

Location coordinates of enterprises of stage 2 are known: $\tau_{1}^{I I}=(0,3 ; 0,4), \tau_{2}^{I I}=(0,8 ; 0,8)$ as well as restrictions for the production capacities of the enterprises: $b_{1}^{I I}=0,2$ and $b_{2}^{I I}=0.8$.

Functions describing expenditures connected with raw material transportation from a supplier with $(x, y)$ coordinates to enterprises with $\tau_{i}^{I}=\left(\tau_{1 i}^{I}, \tau_{2 i}^{I}\right)$ coordinates are set as follows:

$c_{i}^{I}\left(x, y, \tau_{i}^{I}\right)=\sqrt{\left(x-\tau_{1 i}^{I}\right)^{2}+\left(y-\tau_{2 i}^{I}\right)^{2}}, i=\overline{1,4}$.

Expenditures connected with the product transportation from the enterprise of stage $1 \tau_{i}^{I}=\left(\tau_{1 i}^{I}, \tau_{2 i}^{I}\right)$ to the enterprise of stage $2 \tau_{j}^{I I}=\left(\tau_{1 j}^{I I}, \tau_{2 j}^{I I}\right)$ are described in the form of:

$c_{i j}^{I I}\left(\tau_{i}^{I}, \tau_{j}^{I I}\right)=\sqrt{\left(\tau_{1 i}^{I}-\tau_{1 j}^{I I}\right)^{2}+\left(\tau_{2 i}^{I}-\tau_{2 j}^{I I}\right)^{2}}, i=\overline{1, N}, j=\overline{1, M}$.

To simplify, assume that $\rho(x)=1 \forall x \in \Omega$.

It is required to arrange enterprises $\tau_{i}^{I}=\left(\tau_{1 i}^{I}, \tau_{2 i}^{I}\right), i=\overline{1,4}$, partite $\Omega$ area into the zones of raw material accumulation by enterprises of stage 1 and determine the volumes of the product delivery from enterprises of stage 1 to enterprises of stage 2 in such a way to minimize a functional of total expenditures (1) connected with raw material and processed product delivery in terms of (2) - (4) conditions.

Fig. 1 represents the initial data to solve the problem: location of centres of enterprises of stage 1 (circles) and stage 2 (squares). In this context, the following was selected as the initial values of components of a vector function $\lambda(\cdot)$ : 
$\lambda_{i}^{(0)}(x)=\left\{\begin{array}{l}0, i=1,2,3 \\ 1, i=4, \quad \forall x \in \Omega\end{array}\right.$

In other words, it was supposed first that all $x \in \Omega$ points belong to subset $\Omega_{4}$.

To solve the problem, $\Omega$ area was covered by means of the net with $h=0.01$ step. Conditions to terminate calculations were the solution of one of the inequalities:

$\left|I_{l}-I_{l-1}\right| \leq 10^{-6}$,

$\left\|\lambda^{(l)}-\lambda^{(l-1)}\right\| \leq 10^{-6}$.

Following results were obtained with the help of the aforementioned algorithm:

- optimal partition of $\Omega$ area represented in Fig.2;

- optimal transportation volumes (shown in Table 1);

- optimal location of enterprises of stage 1 :

$\tau_{1}^{I^{*}}=(0,82 ; 0,1), \tau_{2}^{I^{*}}=(0,76 ; 0,6), \tau_{3}^{I^{*}}=(0,39 ; 0,27) ;$

- corresponding capacities of enterprises of stage 1 :

$b_{1}^{I}=0,047 ; b_{2}^{I}=0,588 ; b_{3}^{I}=0,365$.

- minimum value of a target functional: $I=1.02127$.

It has been turned out that it is quite sufficient to arrange only three enterprises of stage 1 to meet the requirements of the problem.

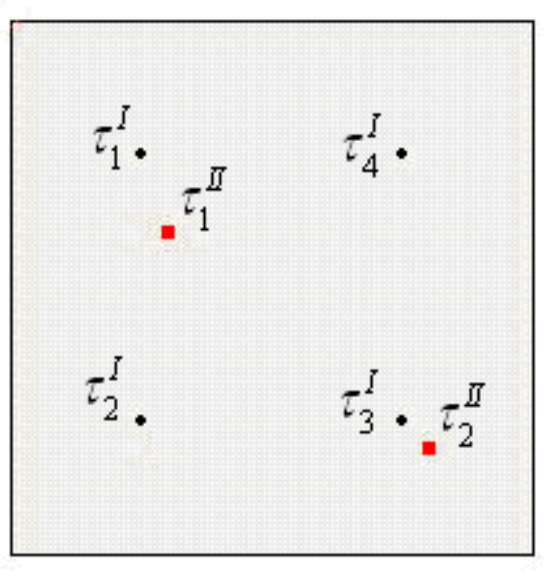

Fig1. Initial data for the model problem

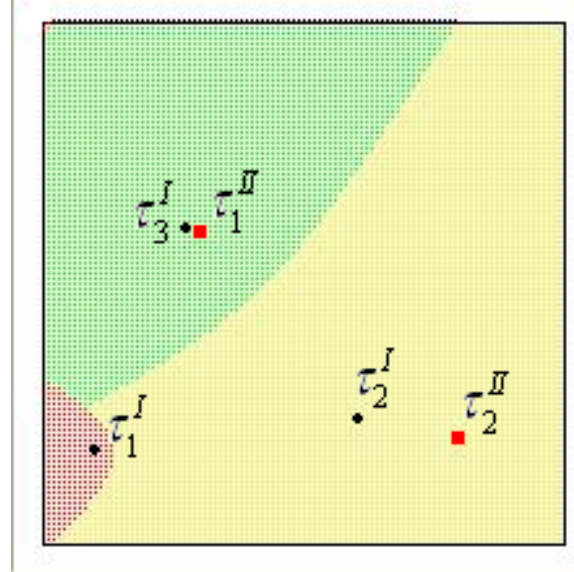

Fig2. Optimal location of centres and the areas partition in the context of the model problem

Demonstrate the importance to consider the additional couplings of stage 1 enterprises being arranged while separating the preset area into the zones of raw material accumulation and their assignment to the corresponding enterprises. To do that, register optimal coordinates of the three centres obtained while solving the model problem, and determine optimal partition of the set, if there are no couplings with enterprises of stage 2 (Fig.3).

Table1. Transportation volumes obtained while the model problem solving

\begin{tabular}{|c|c|c|}
\hline & $\tau_{1}^{I I}$ & $\tau_{2}^{I I}$ \\
\hline$\tau_{1}^{I}$ & 0 & 0.047 \\
\hline$\tau_{2}^{I}$ & 0 & 0.588 \\
\hline$\tau_{3}^{I}$ & 0.2 & 0.165 \\
\hline
\end{tabular}




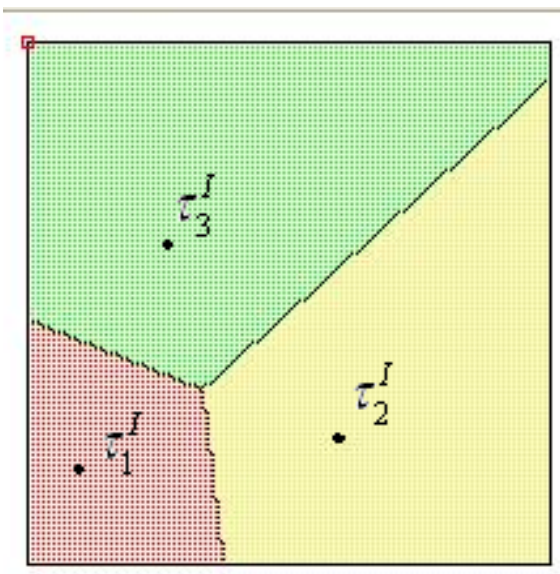

Fig3. The result of OPS solution in the context of registered centres obtained in the process of the model problem solving

The effect of additional couplings between enterprises of the both stages on the optimal partition of the preset area can also be observed in Figures 4 and 5, where optimal partitions of $\Omega$ area are represented. They have been obtained while solving OPS problem with the registered four centres (enterprises of stage 1) without centres of stage 2 and with their availability respectively.

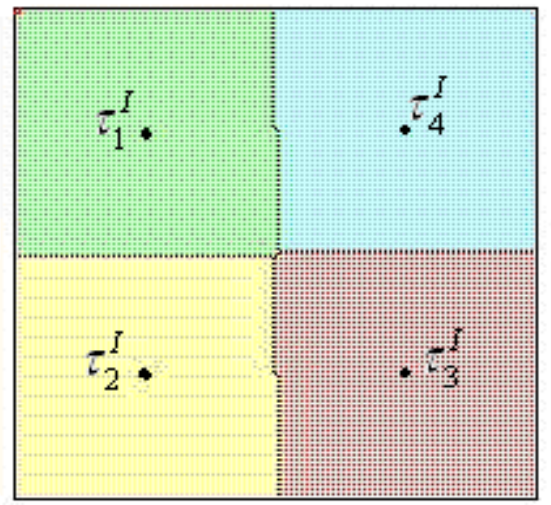

Fig4. Results of OPS solution in the context of registered centres of subsets without restrictions

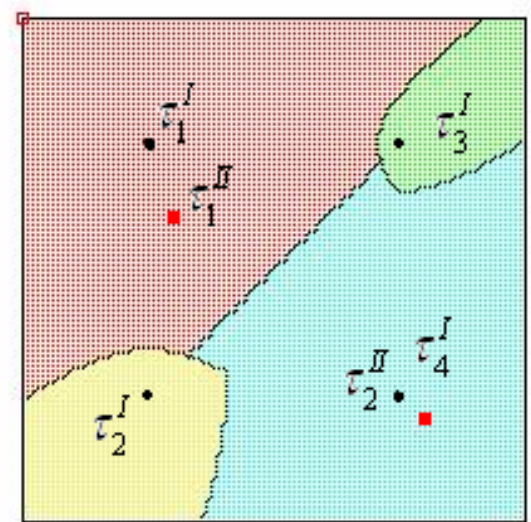

Fig5. Results of OPS AC problem solving in the context of registered centres

Following values of the capacities of enterprises have been obtained in the process of solving standard OPS problem with registered centres:

$b_{1}^{I}=0,2474 ; b_{2}^{I}=0,2426 ; b_{3}^{I}=0,2476 ; b_{4}^{I}=0,2425$,

And values of a target functional: $I=0.5753$.

Following results have been obtained after solving the problem of optimal partition of sets with the additional couplings (OPS AC) in the context of registered centres:

- optimal transportation volumes (shown in Table 2);

- capacities of stage 1 enterprises being equal respectively:

$b_{1}^{I}=0,438 ; b_{2}^{I}=0,119 ; b_{3}^{I}=0,069 ; b_{4}^{I}=0,373$,

- minimum value of a target functional: $I=1.42$.

Table2. Transportation volumes resulting from the solution of model problem 2

\begin{tabular}{|c|c|c|}
\hline & $\tau_{1}^{I I}$ & $\tau_{2}^{I I}$ \\
\hline$\tau_{1}^{I}$ & 0.2 & 0.238 \\
\hline$\tau_{2}^{I}$ & 0 & 0.119 \\
\hline
\end{tabular}




\begin{tabular}{|c|c|c|}
\hline$\tau_{3}^{I}$ & 0 & 0.069 \\
\hline$\tau_{4}^{I}$ & 0 & 0.373 \\
\hline
\end{tabular}

Thus, availability of additional couplings for enterprises being arranged and which effect areas are being determined act significantly on the boundaries between the zones. If there are no such couplings, then the area is divided actually into subsets being equal in their capacities. If such couplings are available, then the grater service zone belongs to the enterprises located closer to stage 2 enterprises; owing to the fact, expenditures connected with the processed product transportation to stage 2 enterprises reduce.

\section{CONCLUSIONS}

Thus, it has been shown that optimal solution of continual two-stage problem of optimal partition of sets with the additional couplings of enterprises being arranged depends upon the finite number of variables being the components of solving the problem of undifferentiated optimization. The importance to involve the additional couplings for enterprises being arranged and which effect areas are taken into consideration has been demonstrated in terms of solving model problems. It has been determined that nonavailability of such couplings stipulates partition of the preset area into the levels according to the subset capacity. If the capacities of stage 1 enterprises depend upon the needs of stage 2 enterprises and the expenditures connected with the product transportation between the production objects should be as less as possible, then the enterprises located closer to stage 2 enterprises obtain a greater operating zone.

\section{REFERENCES}

[1] Gimadi, E.Kh., Effective algorithms to solve multistage problem of location within a chain, Discrete analysis and operational research. October-December 1995. Volume 2, \# 4, pp. 13-31

[2] Kiseliova E.M., Koriashkina L.S., Models and methods to solve continual problems of optimal partition of sets, K. : Naukova dumka, 606 pp., (2013)

[3] Kiseliova E.M., Koriashkina L.S., Us S.A., Theory of optimal partition of sets in the problems of image identification, analysis and system identification, Ministry of Education and Science of Ukraine; National Mining University, D. : NMU, 270, (2015).

[4] Kiseliova, E.M., Shor. N.Z., Continual problems of optimal partition of sets: theory, algorithms, applications: Monograph, K. : Naukova dumka, 564, (2005).

[5] Us, S.A. Stanina, O.D., On the mathematical models of multistage problems of enterprise location, Problems of applied mathematics and mathematical modeling: collection of scientific papers, D.: Publishing house "Lira", Pp.258-268, (2014)

[6] Drezner Z. Hamacher H., Facility Location: Application and Theory, Berlin: Springer, 457, (2001)

[7] Fengqi You Ignacio E. Grossmann, Mixed-Integer Nonlinear Programming Models and Algorithms for Large-Scale Supply Chain Design with Stochastic Inventory Management, Ind. Eng. Chem. Res. 47, 7802-7817, (2008)

[8] Trubin V. Sharifov F., Simple multistage location problem on a treelike network, Cybernetics and Systems Analysis. November-December, 1992, Volume 28, Issue 6, pp 912-917

[9] Yu. Kochetov Bilevel facility location: discrete models and computational methods, Proceedings Of XXXVII Symposium în Operations Research (SYMOPIS-2010), pp 12-16, (2010)

\section{AUTHORS' BIOGRAPHY}

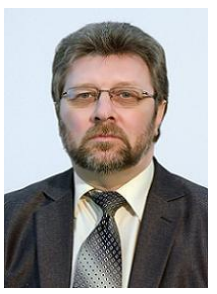

Zelentsov Dmitri, is Doctor of Engineering Science, Head of the Department of Information Systems, Ukrainian State University of Chemical Technology, (Dnipro, Ukraine). Area of scientific interests: modeling behavior of complex systems; artificial intelligence systems; neural networks; genetic algorithm

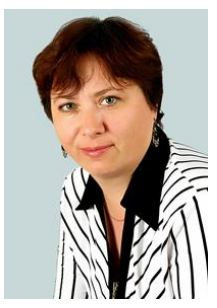

Us Svitlana, is Candidate of Physico-Mathematical Sciences, Professor at the Department of System Analysis and Management, National Mining University, (Dnipro, Ukraine). Area of scientific interests: optimization, optimal partition, continual set partitioning problem, optimal location 


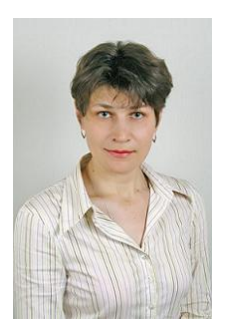

Koryashkina Larysa, is Candidate of Physico-Mathematical Sciences, Associate Professor at the Department of System Analysis and Management, National Mining University, (Dnipro, Ukraine). Area of scientific interests: mathematical models; system analysis; systems dynamics; continual set partitioning problem

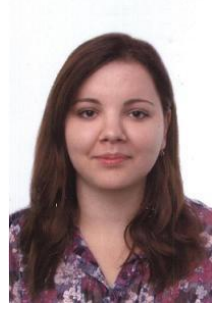

Stanina Olga, is a Teaching Assistant of Department of Information Systems, Ukrainian State University of Chemical Technology, (Dnipro, Ukraine). Area of scientific interests: optimization, continual set partitioning problem, locationallocation problem

Citation: Stanina O, et al,(2017). Solving Continual Two-Stage Problems of Optimal Partition of Sets, International Journal of Research Studies in Computer Science and Engineering (IJRSCSE), 4(4), pp.72-80, DOI: http://dx.doi.org/10.20431/2349-4859.0404009

Copyright: (C) 2017 Stanina O, This is an open-access article distributed under the terms of the Creative Commons Attribution License, which permits unrestricted use, distribution, and reproduction in any medium, provided the original author and source are credited 